\title{
El servicio de referencia en la biblioteca digital
}

\author{
Georgina Araceli Torres Vargas * \\ María Del Rocío Sánchez Avillaneda **
}

Artículo recibido:

1 de octubre de 2004.

Artículo aceptado:

14 de marzo de 2005.

\section{RESUMEN}

El servicio de referencia se muestra como uno de los servicios más importantes de la biblioteca digital. A grosso modo, se presenta en el artículo un bosquejo histórico del surgimiento del servicio, su concepto y sus características, así como la aparición de dicha práctica, pero ahora inmersa en un nuevo ambiente -el digital-y puntualizamos las características de este fenómeno.

Palabras clave: Servicio de referencia; Servicio de referencia digital.

* Centro Universitario de Investigaciones Bibliotecológicas de la UNAM, México. gatv@servidor.unam.mx

** Instituto de Investigaciones en Matemáticas Aplicadas y Sistemas de la UNAM, México.marsa@leibniz.iimas.unam.mx

INVESTIGACIÓN BIBLIOTECOLÓGICA, VOL.19, NÚM.39, JULIO/DICIEMBRE, 2005 
ABSTRACT

Reference service in the digital library

Georgina Araceli Torres-Vargas and

María del Rocío Sánchez-Avillaneda

The reference service comes to light as one of the most important practices of the digital library. Essentially, the paper draws a historical sketch about the emergence of the service, its conceptualization and its characteristics, as well as the advent of this practice, but now immersed in a new environment_the digital. We intend to show the characteristics of this phenomenon.

Keywords: Reference services; Digital reference services.

\section{INTRODUCCIÓN}

T a biblioteca digital o virtual es un sistema de información en red, que les Lofrece a sus usuarios contenidos y servicios digitales cuya información y medios de comunicación se encuentran en servidores distribuidos en diferentes latitudes del mundo. En esta noción de biblioteca los servicios son resultado del manejo de la información digital a través de tecnologías de la comunicación y de la información, lo que le otorga características distintas a las de los servicios que se ofrecen en la biblioteca convencional.

Es así como los servicios digitales de información se presentan hoy como un campo fértil para analizar la problemática y retos que han surgido con la intención de derivar propuestas de servicios idóneos.

Existen, sobre todo en lengua inglesa, diversos trabajos en los que se aborda lo referente a los servicios que ofrece la biblioteca digital, y en particular el servicio de referencia. ${ }^{1}$ Sin embargo pese a lo numeroso de este tipo de estudios, en casi todos los casos se ejemplifica a partir de los intentos que se están haciendo para poner en marcha servicios de consulta. De esta forma muchos artículos enfocan al trabajo de referencia mediante la comparación de paquetes de software, el estudio sobre el desempeño del bibliotecario de referencia en línea, o el análisis de las necesidades de información, entre otras

1 Son numerosos los trabajos en que se aborda el tema de servicio de referencia digital. Basta ver la bibliografía que muestra Sloan Bernie (Digital reference services bibliography. Disponible en:

http://www.lis.uiuc.edu/\%7Eb-sloan/digiref.html), en donde lista más de 700 artículos. 
temáticas encaminadas en su gran mayoría a describir qué tan buen o mal camino siguen diversas bibliotecas en el desarrollo del servicio de referencia a distancia. $^{2}$

Por eso se puede afirmar que casi no existen trabajos que desde una perspectiva teórica permitan hacer generalizaciones de los aspectos y problemáticas que se relacionan con el servicio de referencia.

Como casi todos los aspectos de la biblioteca digital se abordan desde una visión puramente empírica, esto provoca la implementación de acciones tendientes a resolver problemas inmediatos en tiempos y espacios particulares, lo que impide el análisis de la situación desde una óptica amplia.

El presente artículo muestra algunos de los elementos que pueden intervenir en el momento mismo en que se diseñan y ofrecen servicios de referencia en la biblioteca digital.

\section{EL SERVICIO DE REFERENCIA EN LA BIBLIOTECA CONVENCIONAL}

Para abordar el análisis del servicio de referencia en la biblioteca digital es necesario revisar brevemente cuáles han sido los aspectos históricos y conceptuales del servicio de referencia en la biblioteca convencional (o tradicional), con el propósito de estudiar una diferente modalidad del servicio; por eso se presentan algunas aportaciones de los autores clásicos que han tratado el tema.

El concepto más antiguo del trabajo de referencia moderno le es atribuido a Samuel Swett Green en su trabajo titulado "Personal relations between librarians and readers" publicado en 1876, documento que refleja la defensa que hace Swett sobre la importancia del servicio personalizado y la orientación en la biblioteca. ${ }^{3}$ Hasta ese momento las bibliotecas sólo se concentraban en adquirir y organizar materiales, y se esperaba que los usuarios fueran autónomos en su búsqueda de información. En este contexto cabe recordar que los servicios bibliotecarios surgieron debido a la preocupación que existía por el registro de los documentos y, principalmente, por la aparición del servicio de préstamo y consulta a partir de la sistematización de materiales que conformaban las colecciones. ${ }^{4}$

2 Steve Coffman. To chat or not to chat- taking another look at virtual reference, Part 1 http://www.infotoday.com/searcher/jul04/arret_coffman.shtml

3 Samuel Swett Green (1876). "Personal relation between libraries and readers", en American Library Journal, vol. 1, p. 74-81.

4 Georgina Araceli Torres Vargas (2003). "Hacia un modelo de servicios en la biblioteca digital”, en Investigación Bibliotecológica: archivonomía, bibliotecología e información, vol. 17 , no. 35 , p. 35 . 
Por su parte, Bopp menciona que las ideas de Green ya contemplaban las tres funciones básicas del servicio de referencia actual: información, guía e instrucción, y vuelve a afirmar que dichas actividades se llevaban a cabo desde el momento en que el usuario entraba a la biblioteca en búsqueda de información.

El surgimiento de esta actividad bibliotecaria se manifestó a través de las necesidades de información que tenía el usuario, es decir desde la aparición de las bibliotecas el servicio de pregunta/respuesta se hizo presente con la pretensión de que, por un lado, el usuario pudiera localizar información y, por el otro, el bibliotecario le proporcionara ayuda a quien se la solicitara. En este sentido el servicio de referencia se ha convertido actualmente en un componente indispensable de los servicios bibliotecarios que brinda cualquier tipo de biblioteca. La ALA menciona que

el servicio de información en la biblioteca considera una variedad de formas incluyendo la asistencia personal directa, directorios, señales, intercambio de información, servicio de alerta, diseminación selectiva de información en anticipación a las necesidades e intereses de los usuarios, y el acceso a la información electrónica. ${ }^{5}$

Así pues, el servicio de referencia resuelve cuestiones de respuesta inmediata y sencilla a través del contacto personal entre usuario/bibliotecario, y su principal objetivo es "conseguir que todas las informaciones requeridas sean satisfechas", de tal manera que la biblioteca cuenta con un espacio muy bien definido y con personal especializado en la búsqueda de información, así como una colección útil y una infraestructura adecuada que refuerza el desarrollo de sistemas sencillos para recibir consultas. ${ }^{6}$

Por otra parte, Lifeng $\operatorname{Han}^{7}$ menciona que el servicio de referencia presenta tres variedades:

1. Servicio de información: el cual considera varios tipos de servicios que van desde una simple provisión de datos (direcciones, nombres, números de teléfonos) hasta el servicio de búsquedas bibliográficas sobre un tema, préstamo interbibliotecario, entrega de documentos, y todos aquellos que están destinados a ofrecer información básica, específica y/o general.

2. Orientación: que incluye los servicios consultivos de los usuarios hacia el bibliotecario a través de preguntas, o bien la aplicación de la

5 ALA. (2000), "Guidelines for Information Services", [En línea] Disponible en:

http://www.ala.org/ala/rusa/rusaprotools/referenceguide/guidelinesinformation.htm

6 José Antonio Merlo Vega (2000). "El servicio bibliotecario de referencia", en Anales de documentación, no. 3, p. 95.

7 Lifeng Han (2003) "Information and reference services in the digital library", en Information services \& use, vol. 23, p. 251. 
biblioterapia como una técnica de autoestima y ayuda psicológica a través de los libros y la lectura -la biblioterapia se practica principalmente en bibliotecas de hospitales, bibliotecas y penitenciarias, entre otras-, y asesoría general.

3. Instrucción: servicio que se puede ofrecer a usuarios individuales o en grupo, y que puede consistir en una simple orientación sobre el uso de la biblioteca, las fuentes de información y/o los servicios. Al respecto, es notable considerar que el tipo y número de fuentes son ahora mucho más numerosos y complejos, principalmente debido a la incursión en fuentes electrónicas, por lo que la instrucción jugará en esto un papel de vital importancia.

A partir de la primera mitad del siglo XX los conceptos y prácticas del trabajo de referencia se fueron ampliando y desarrollando significativamente, de tal manera que las formas de proporcionar el servicio y las variedades de los tipos de materiales también fueron cambiando a la par que los usuarios demandaban información sin que importara el tipo de soporte, tiempo o lugar. Desde esta perspectiva puede considerarse que el servicio de referencia implica una atención personalizada por parte del bibliotecario hacia los usuarios que buscan información; tan es así que la atención es la esencia de este tipo de servicio y el papel fundamental del bibliotecario de referencia. Esta atención personalizada no se elimina en el medio digital, pero adquiere otras características, veamos cuáles.

\section{EL SERVICIO DE REFERENCIA DIGITAL}

Joan M. Wasik manifiesta que el servicio de referencia digital comenzó a ofrecerse para complementar los servicios tradicionales proporcionando ayuda en un ambiente electrónico. Uno de los primeros servicios en línea fue el ERAS (Electronic Access to Reference Service) lanzado por la Universidad de Maryland en Baltimore en 1984, y aunque los primeros esfuerzos se basaron en el uso de correo electrónico éstos recibieron poca atención por parte de los usuarios; sin embargo poco a poco el servicio digital proliferó y llegó a ser cada vez más popular, al grado que surgió el servicio AskERIC en 1992. Actualmente los servicios de referencia digital se han convertido en recursos importantes y eficaces para resolver las necesidades de información de muchos usuarios. $^{8}$

8 J. M Wasik. "Building and maintaining digital reference services". [En línea] Disponible en: http://www.ericfacility.net/ericdigests/ed427794.html 
Desde la perspectiva de Lankes, el término de referencia digital surge en dos momentos de las necesidades de información; el primero se encuentra en la práctica de la biblioteca tradicional y de las ciencias de la información y ahí el papel del referencista en un ambiente digital comienza con el apoyo del correo electrónico que amplia la función tradicional del servicio básico de la biblioteca hacia un servicio de referencia mucho más especializado y rápido a través de una computadora; mientras que el segundo momento y la mayor contribución a esta nueva forma de servicio es la categoría de Internet conocida como AskA services o sitios expertos de preguntas/respuestas.

Por ello Lankes considera que el término referencia digital se refiere a la red de expertos, a la intermediación y a los recursos puestos a disposición de una persona que busca respuestas en un ambiente en línea. También se conoce esto bajo los términos de referencia virtual, referencia en tiempo real, y referencia en vivo, ${ }^{9}$ considerando que el uso del correo electrónico, chat, los mensajes instantáneos y las videoconferencias, permiten una comunicación inmediata. Este autor señala también que todas estas tecnologías tienen un propósito fundamental: proporcionar mediación entre el ser humano y la información en línea. En este sentido, el concepto central consiste en el uso de Internet y un software especial que facilite la comunicación entre el usuario/ referencista en un espacio diferente y en el tiempo que se desee.

En agosto de 2002, en Harvard, se llevó a cabo un simposio de investigación relacionado con el servicio de referencia digital ${ }^{10} \mathrm{y}$ de éste hemos podido rescatar algunas de las definiciones presentadas, entre ellas la de Whitlatch, quien manifiesta que los servicios de referencia digital están basados en Internet y que proporcionar información a los usuarios requiere de personas expertas o intermediarios; por su parte Pomerantz considera que el servicio de referencia digital provee a los usuarios respuestas a sus preguntas realizadas en un ambiente por computadora.

Una tercera opinión presentada por Marylin D. White, define al servicio de referencia digital como un servicio de acceso de información a través de medios electrónicos.

Por su parte, el ODLIS (Online Dictionary for Library and Information Science) define al servicio de referencia digital (digital reference) como aquél que proporciona información a través de Internet, generalmente vía correo electrónico, mensajería inmediata (charla) o a través de preguntas contestadas por los bibliotecarios; y lo considera sinónimo de referencia electrónica

9 R. David Lankes (2004). "Digital reference", en Encyclopedia of Library and Information Science, New York, Marcel Dekker, pp. 1-3.

10 R. David Lankes, A. Goodrum \& S. Nicholson (2002). The Digital Reference Research Agenda, Chicago, IL., Publications in Librarianship, Association of College \& Research Libraries. 
(e-reference), referencia en línea (online reference) y referencia virtual (virtual reference). ${ }^{11}$

Otra definición es la que presenta el VRD (Virtual Reference Desk: proyecto financiado por el Departamento de Educación de EUA y dedicado a la investigación sobre referencia digital), el cual considera que la referencia digital o AskA son aquellos servicios de Internet, basados en preguntas y respuestas, que conectan a los usuarios con expertos; los servicios de referencia digital utilizan Internet para conectar personas con personas que puedan contestar preguntas y apoyar el desarrollo de habilidades. ${ }^{12}$

Por su parte, Lifeng Han menciona que los servicios de referencia digital son un término que representa a aquellos servicios proporcionados a través de medios electrónicos y que hacen uso de software asincrónico (correo electrónico, y otras formas del web) y sincrónico (sistema en tiempo real como el "chat"). En un sentido más estricto, la referencia digital se utiliza generalmente como un sinónimo de referencia electrónica en tiempo real. De esta manera, el autor señala que los servicios de referencia tradicional se realizan cara a cara o en un contacto directo a través del teléfono o fax dentro de la biblioteca; mientras que con el servicio de referencia digital los usuarios pueden tener acceso a los servicios a pesar del tiempo y la distancia, siendo éste último un servicio que les brinda más alternativas y flexibilidad a los usuarios. ${ }^{13}$

Para Wasik nuestro tema es un conjunto de servicios apoyados en Internet y basados en preguntas y respuestas que conectan a los usuarios con expertos en una variedad de temas. Adicionalmente a las preguntas que contestan los expertos (referencistas) pueden también proveer y remitir a los usuarios a otras fuentes en línea.

Hasta aquí, a través de las consideraciones hechas por los diferentes autores, se puede identificar que para ofrecer un eficaz servicio de referencia digital es imprescindible echar mano de la tecnología (software y hardware) pues sólo así se puede tener comunicación inmediata entre el referencista y el usuario. El Cuadro 1 describe algunos de los tipos de sistemas o dispositivos a través de los cuales la biblioteca digital puede proporcionar servicios de referencia.

11 Joan M. Reitz. "Digital reference". ODLIS: Online Dictionary for Library and Information Science, [En línea] Disponible en:

http://lu.com/odlis/odlis_r.cfm

12 "The Virtual Reference Desk", [En línea] Disponible en: http://www.vrd.org 
Cuadro 1. Tipología de los sistemas y recursos utilizados para proporcionar el servicio de referencia

\begin{tabular}{|c|c|}
\hline Sistema de comunicación & Características \\
\hline Correo electrónico & $\begin{array}{l}\text { Recursos que le permiten al usuario enviar al referencista un mensaje } \\
\text { con una pregunta sobre la información que requiere, y en donde a su } \\
\text { vez el referencista provee información a través del mismo medio o } \\
\text { bien vía telefónica, fax, etcétera. Es de carácter asíncrono ya que no } \\
\text { requiere la intervención del emisor y receptor al mismo tiempo. }\end{array}$ \\
\hline Chat & $\begin{array}{l}\text { Recurso que le permite al usuario y al referencista mantener una } \\
\text { charla a través de mensajes breves (pregunta/respuesta) en tiempo } \\
\text { real, de tal manera que intercambian información a través de una se- } \\
\text { rie de mensajes cortos hasta conseguir la petición del usuario. Es de } \\
\text { tipo sincrónico. }\end{array}$ \\
\hline Formularios-web & $\begin{array}{l}\text { Herramientas que ayudan a clarificar la solicitud del usuario, donde el } \\
\text { referencista le pide al usuario que llene un formulario anexo a su peti- } \\
\text { ción en donde especifique más detalles sobre su solicitud de informa- } \\
\text { ción. Se caracteriza por ser una herramienta de tipo asíncrono. }\end{array}$ \\
\hline Mensajería instantánea & $\begin{array}{l}\text { Es una de las formas síncronas más populares de comunicación por } \\
\text { computadora y funciona como un servicio personalizado que propor- } \\
\text { ciona interacción inmediata con el referencista. }\end{array}$ \\
\hline Realidad virtual & $\begin{array}{l}\text { La tecnología de realidad virtual permite hacer simulaciones de diver- } \\
\text { sos escenarios. Para el servicio de referencia, la realidad virtual per- } \\
\text { mite "llevar" al usuario al interior de la biblioteca y, a manera de visita } \\
\text { guiada, mostrarle los espacios, la disposición de las colecciones, y } \\
\text { los catálogos y servicios que se prestan. }\end{array}$ \\
\hline Videoconferencia & $\begin{array}{l}\text { Servicio multimedia que permite la interacción entre dos o más gru- } \\
\text { pos de personas, su función básica consiste en la interconexión me- } \\
\text { diante sesiones interactivas donde todos pueden verse y hablarse en- } \\
\text { tre sí. Es de tipo sincrónico. }\end{array}$ \\
\hline
\end{tabular}

Estas nuevas tecnologías vinculadas a Internet presentan tanto convenientes como limitantes para el servicio de referencia, tal es el caso del uso del correo electrónico (E-mail) que en comparación con el correo convencional es de carácter inmediato y su tiempo de envío es mucho más corto, otra ventaja adicional es el bajo costo en relación con una llamada telefónica de larga distancia, y se pueden hacer envíos a muchos destinos al mismo tiempo incluyendo archivos adjuntos (attachments) de diversos formatos. En cuanto a la obtención del servicio se debe contar con un proveedor de acceso a Internet, lo cual implica requerimientos de tipo económico y un equipo de cómputo; pero a la vez existe la limitante de no poder recibir todos los mensajes por el espacio insuficiente al que se tiene derecho de acuerdo con las políticas del proveedor del servicio. 
En cuanto al uso del Chat éste permite hacer todos los ajustes necesarios con el propósito de evitar cualquier mal entendido al hacer la petición de la información, lo que además instaura una entrevista en la que se intercambian una serie de mensajes cortos que permiten obtener una idea más clara de lo que el usuario requiere y lo que el referencista ofrece. Como contraparte la charla presenta algunas desventajas que es necesario mencionar: limita el uso del envío de documentos en formatos como pdf, Word, ligas y otros, en cuyo caso el usuario tendría que abrir otra sesión, en otro tiempo y espacio, para evitar desconectarse de la charla. A diferencia de la comunicación por voz regular, en este servicio se invierte más tiempo al tener que escribir el texto de envío de pregunta/respuesta y por ende existe mayor probabilidad de hacer errores mecanográficos. Y por lo que toca a la rapidez de la respuesta, el usuario fácilmente pierde la paciencia al tener que esperar la información, ya que por lo general está acostumbrado a que el servicio sea no sólo eficiente sino también inmediato. ${ }^{14}$

Los formularios web permiten obtener información categorizada y específica de lo que el referencista requiere saber, mientras que para el usuario es mucho más sencillo y atractivo, y sirve como guía tanto para el emisor como para el receptor del mensaje. Este tipo de recurso herramienta requiere de la planeación y diseño de la hoja web más el uso de algún lenguaje de máquina como puede ser HTML (HyperText Markup Language).

En relación a la Mensajería instantánea (MI) éste resulta un servicio mucho más rápido que el correo electrónico y mejor que una llamada telefónica, y tiene además la ventaja de adjuntar archivos y tener acceso inmediato a sitios web sin desconectarse de la sesión. Otra característica más es que resulta menos probable que la línea de comunicación esté ocupada que en una conversación telefónica común. La mensajería instantánea tiene las mismas ventajas que el Chat y, mejor aún, ofrece otros servicios adicionales que permiten enviar URL's, imágenes y recibir preguntas y respuestas breves o extensas. ${ }^{15}$ Una de las desventajas es que existe filtración de otros usuarios en anonimato que no están dados de alta, lo que provoca inseguridad en el sistema.

La tecnología de realidad virtual sólo cumple la función de familiarizar al usuario con la biblioteca tal y como ésta es físicamente. De alguna forma suple al bibliotecario en la tarea de hacer una visita guiada por la biblioteca.

Por otra parte, la gran ventaja del uso de la videoconferencia consiste en reunir grupos de interés ubicados en diferentes lugares geográficos; sin embargo

14 Trix Bakker (2002). "Virtual reference services: connecting users with experts and supporting the development of skills". Liber Quarterly, vol. 12, p. 124-137.

15 Christina M. Desai (2003). "Instant messaging reference: how does it compare?", en The

Electronic Library, vol. 21, no. 1, p.21-30. 
una de las desventajas principales es el alto costo de los equipos destinados a este servicio, incluyendo la renta de canales de comunicación, los servicios de mantenimiento y, obviamente, el personal calificado encargado de realizar las diversas tareas que implica la implementación de este tipo de comunicación.

Retomando algunas consideraciones conceptuales hechas por los diferentes autores, se pueden identificar dos grupos de opiniones en torno del papel que habrá de desempeñar el referencista en el entorno digital:

a) Uno en donde el bibliotecario de referencia adquiere el papel de educador, y quien ayudará a que el usuario sepa utilizar las tecnologías disponibles, así como a utilizar y diseñar estrategias de búsqueda por sí mismo.

Desprendida de esta tendencia, se considera en el medio digital que el desarrollo de habilidades informativas es esencial, sobre todo en el contexto de la educación a distancia, en donde el estudiante requiere contar con aquellos elementos que le permitan utilizar al máximo la biblioteca digital. ${ }^{16}$

En el caso de la educación a distancia, la biblioteca digital deberá tener a disposición del alumno las obras de consulta digitales, así como bases de datos y demás herramientas que le permitan localizar la información puntual que requiere, además de tutoriales que ayuden en el aprendizaje sobre el manejo de las fuentes digitales de consulta. Al bibliotecario de consulta le corresponderá mantener estos recursos de información al día y en concordancia con las necesidades de los usuarios.

b) Otro grupo de opiniones muestra al referencista como aquel intermediario entre la información y el usuario, que de manera personalizada busca, recupera y entrega la información que el usuario requiere. Es el experto que conoce y maneja las fuentes de información, recupera los datos que se solicitan vía correo electrónico, etcétera, y remite todo al usuario final.

En este caso es el bibliotecario quien debe encontrar los caminos y medios para satisfacer a todos los usuarios que le plantean una pregunta. En

$16 \mathrm{Al}$ respecto Jesús Lau menciona que en los procesos orientados al aprendizaje, se requieren servicios informativos que apoyen en el desarrollo de métodos de autoestudio, autoaprendizaje, investigación, experimentación, trabajo en equipo, etcétera. De aquí que sea necesario el desarrollo de habilidades informativas en el estudiante. $C f r$. Jesús Lau. Información: insumo básico del aprendizaje, pp. 51-78, en Los grandes problemas de la información en la sociedad contemporánea: Memoria del XIX Coloquio Internacional de Investigación Bibliotecológica y de la Información 28,29 y 30 de agosto de 2001/Compiladoras Martha Alicia Añorve Guillén, Elsa M. Ramírez Leyva. México: UNAM, CUIB, 2002. 
esta última tendencia, cada necesidad de información se convierte en responsabilidad del referencista, no del usuario. ${ }^{17}$

Ante tal escenario la práctica del servicio de referencia presenta nuevos retos, ya que ahora se proporciona a través de un acceso remoto con usuarios remotos que no conocemos, e incluso permite la consulta de materiales que no son propios de la colección de la biblioteca a través de medios electrónicos. Al respecto, Shelda Debowski ${ }^{18}$ define al usuario remoto como aquél que tiene acceso a los servicios bibliotecarios sin realmente entrar por las puertas de la biblioteca, de ahí que su planteamiento se justifica cuando sostiene que la naturaleza de los usuarios de la biblioteca está presentando cambios notables, y que la forma de ayudar al usuario es distinta en comparación a como se venía realizando. Asimismo la autora señala que la biblioteca deberá empezar a rediseñar la forma de proporcionar los nuevos servicios para los nuevos usuarios, en el entendido de que la relación con un usuario local es totalmente directa.

Si bien a simple vista esta forma de ofrecer y recibir el servicio de referencia pareciera más impersonal que nunca, no hay que perder de vista que el bibliotecario estará en todo momento tratando de responder a un conjunto de necesidades específicas aun cuando su labor la desarrolle a distancia, sea a través del correo electrónico o del chat. La relación usuario-bibliotecario no es cara a cara, pero no por ello es impersonal.

Por otro lado se piensa que el servicio de referencia remoto le ofrece al usuario la ventaja de obtener la información que desea a cualquier hora que la solicite. Sin embargo en la práctica se observa que los costos, tanto de personal como de equipo, para dar servicio las 24 horas del día son muy altos. Lo usual sigue siendo el establecimiento de horarios para el préstamo del servicio, por lo que la inmediatez es relativa. ${ }^{19}$

Estos son algunos de los aspectos que caracterizan al servicio de consulta en la biblioteca digital, pero no puede decirse que ya todo esté dado en esta materia, pues en la actualidad se sigue discutiendo en el medio bibliotecológico internacional cuál podrá ser el futuro de este servicio. Otra situación que se está dando en los numerosos congresos y artículos que analizan estas temáticas, es el abordaje de aspectos particulares sobre el servicio de referencia digital, de manera que se puede ver cierta especialización. Por ejemplo,

17 Introducción general al servicio de consulta: libro de texto para el estudiante de bibliotecología y manual para el bibliotecario de consulta/Richard E. Bopp, Linda C. Smith; trad. Carlos Compton García. - México: UNAM, CUIB, 2000. - p. 15.

18 Shelda Debowski (2003). "Service to remote library users", en Enciclopedia of library and information science, 2a ed., Marcel Dekker, New York, pp.226-235.

19 Como ejemplo de ello, se puede revisar el documento: Delivering Library Services at a Distance: A Case Study at the University of Washington/Angela Lee. Disponible en: http://www.westga.edu/ library/jlsde/jlsde2.1.html (Fecha de consulta: 25-sep-2004). 
se habla del servicio de consulta en la educación a distancia, así como en los diversos tipos de bibliotecas (académicas, públicas,etcétera).

\section{CONCLUSIONES}

A manera de conclusiones se puede decir que el concepto de servicio de referencia sigue siendo vigente en la biblioteca digital. El servicio de referencia digital se puede definir como la conexión realizada entre usuario-máquinareferencista, a través de la injerencia de medios electrónicos en un ambiente digital, con obras de consulta digitales.

Si bien la asistencia que se da es a distancia, sigue siendo personalizada; sobre todo cuando el bibliotecario mantiene una charla con el usuario vía correo electrónico o vía chat, para definir el tipo de información que requiere.

En el caso de optar por el préstamo de un servicio de referencia en donde el usuario sea quien localice su información, el bibliotecario requiere igualmente conformar una colección de referencia digital acorde con las necesidades del grupo de usuarios al que sirve. Ante esta modalidad, el bibliotecario se encarga de mantener contacto a distancia con el usuario para ayudarlo con las posibles dificultades que éste pudiera tener al momento de utilizar los recursos de información. Y es además bajo esta óptica, como el bibliotecario de referencia deberá ser experto en el manejo de la información digital, así como un conocedor del mercado de programas y demás material de apoyo, para que el alumno de educación a distancia aprenda a utilizar la biblioteca digital.

Visto así, el referencista sigue siendo un conocedor de las fuentes de información, pero es además un educador preocupado por ayudar al desarrollo de habilidades informativas en el usuario y a que se actualice en el manejo de aquellas tecnologías de información y comunicación que le pueden ser útiles. Como puede verse, en esencia, el concepto y las funciones del bibliotecario no han cambiado sino que se han ampliado.

Hoy se discute mucho sobre el desarrollo de la nueva faz del servicio de referencia y de la relación que debe o debería existir entre el bibliotecario y los usuarios. Hasta el momento lo único que se puede afirmar es que el servicio de referencia remoto permite la obtención puntual de información sin necesidad de tener que desplazarse físicamente a la biblioteca, aspecto que representa una gran y obvia ventaja.

Sin embargo falta solucionar adecuadamente problemas como el de los costos, para que la inmediatez de la recuperación y entrega de información se haga realidad. 
Pese a las limitantes que actualmente tiene el servicio de referencia en la biblioteca digital se considera una gran promesa y a la vez un reto para el bibliotecario de consulta. Pero sobre todo representa un tema de estudio que requiere de un análisis al interior de la bibliotecología, sobre todo en razón de que existe la necesidad de replantear algunos de los servicios de información.

\section{OBRAs CONSUltadAs}

ALA. (1983), Glossary of library and information science, ed. Heartsill Young, ALA, Chicago.

ALA. (2000), "Guidelines for information services", [En línea] Disponible en:

http//www.ala.org/rusa/stnd_consumer.html

Bakker, Trix. (2002), "Virtual reference services: connecting users with experts and supporting the development of skills", en Liber Quarterly, vol. 12, p. 124-137.

Bopp, R. E. (2001), "History and varieties of reference services", en Reference and information services: an introduction, $3 \mathrm{rd}$ ed., Libraries Unlimited, Englewood.

Bunge, Charles A. (2001), "Vision and values: touchstones in times of change", en Bopp, R. E. Reference and information services: an introduction, 3rd ed., Libraries Unlimited, Englewood.

Buonocore, D. (1976), Diccionario de bibliotecología; términos relativos a la bibliología, bibliografía, bibliofilia, biblioteconomía, archivología, documentación, tipografía y materias afines, Marymar, Buenos Aires.

Debowski, Shelda. (2003), "Service to remote library users", en Encyclopedia of library and information science, $2 \mathrm{a}$ ed. Edited by Miriam A. Drake, Marcel Dekker, New York, pp. 2626-2635.

Delivering Library Services at a Distance: A Case Study at the University of Washington / Angela Lee. Disponible en: http://www.westga.edu/ library/jlsde/jlsde2.1.html (Fecha de consulta: 25-sep.-2004)

Desai, Christina M. (2003). "Instant messaging reference: how does it compare?", en The Electronic Library, vol. 21, no. 1, p.21-30.

Green, Samuel Swett. (1876), "Personal relation between libraries and readers", en American Library Jornal, vol. 1, pp. 74-81.

Han, Lifeng and Anne Goulding. (2003), "Information and reference services in the digital library", en Information services $\mathcal{E}$ use, vol. 23, pp. 251-262.

Harrod, Leonard Montague. (1977), The librarian's glossary of terms used in librarianship, documentation and the book crafts, Andre Deutsch, Great Britain. 
Introducción general al servicio de consulta: libro de texto para el estudiante de bibliotecología y manual para el bibliotecario de consulta I Richard E. Bopp, Linda C. Smith; trad. Carlos Compton García. - México: UNAM, CUIB, 2000.

Lankes, R. David. (2004), "Digital reference", en Enciclopedia of Library and Information Science, p. 1.

Lankes, R. David, Goodrum, A., \& Nicholson, S. (2002), The Digital Reference Research Agenda. Chicago, Publications in Librarianship, Association of College \& Research Libraries, Illinois.

Lau, Jesús. "Información: insumo básico del aprendizaje”. - pp. 51-78, en Los grandes problemas de la información en la sociedad contemporánea : Memoria del XIX Coloquio Internacional de Investigación Bibliotecológica y de la Información 28,29 y 30 de agosto de 2001 / Compiladoras Martha Alicia Añorve Guillén, Elsa M. Ramírez Leyva. - México: UNAM, CUIB, 2002.

Merlo Vega, José Antonio. (2000), "El servicio bibliotecario de referencia”, en Anales de documentación, no. 3, pp. 93-126.

Pomerantz, J. (2003), "Integrating Digital Reference Service into the Digital Library Environment", en The Digital Reference Research Agenda. Lankes, R. David, Goodrum, A., \& Nicholson, S. (Eds.). Publications In Librarianship, Association of College \& Research Libraries; Chicago, IL.

Reitz, Joan M. "Reference services”. ODLIS: Online Dictionary for Library and Information Science, [En línea] Disponible en: http://lu.com/odlis/odlis_rcfm

Torres Vargas, Georgina Araceli. (2003), "Hacia un modelo de servicios en la biblioteca digital", en Investigación Bibliotecológica: archivonomía, bibliotecología e información, vol. 17, no. 35, pp. 35 46.

The Virtual Reference Desk, [En línea] Disponible en: http://www.vrd.org

Wasik, J. M. "Building and maintaining digital reference services". [En línea] Disponible en: http://www.ericfacility.net/ericdigests/ed427794.html

Whitlatch, J. (2003), "Policies for Digital Reference", The Digital Reference Research Agenda. Lankes, R. David, Goodrum, A., \& Nicholson, S. (Eds.). Publications In Librarianship, Association of College \& Research Libraries; Chicago, IL. 